\title{
Remediation of a constitutive model for ceramic composite laminates
}

\author{
Varun P. Rajan*, Frank W. Zok \\ Materials Department, University of California, Santa Barbara, CA 93106, United States
}

\section{A R T I C L E I N F O}

\section{Article history:}

Received 20 January 2013

Received in revised form 11 May 2013

Accepted 18 May 2013

Available online 28 May 2013

\section{Keywords:}

A. Ceramic-matrix composites (CMCs)

B. Mechanical properties

C. Computational modelling

C. Finite element analysis

\begin{abstract}
A B S T R A C T
Constitutive models for fiber-reinforced ceramic-matrix composites (CMCs) are needed to enable implementation of these materials in future engineering systems. One such constitutive model, developed by Genin and Hutchinson [1], is based on a phenomenological description of the inelastic response of CMC laminates. Although the model has found some utility in elucidating the role of inelasticity in stress redistribution around strain concentrating features, we find that, in some instances, finite element analyses based on this model exhibit numerical convergence problems. In the present study, we demonstrate both analytically and by finite element analyses that, for certain anisotropic laminates, these numerical issues stem from the fact that the model formulation is unstable. Additionally, we propose and assess modifications to the formulation that mitigate these problems yet retain the positive features of the original model. The expectation is that the modifications will enable broader utilization of the model within the engineering design community.
\end{abstract}

(c) 2013 Elsevier Ltd. All rights reserved.

\section{Introduction}

Genin and Hutchinson [1] developed a phenomenological constitutive model for ceramic matrix composite (CMC) laminates with balanced fiber architectures subject to plane-stress loadings. The model is closely analogous in many respects to deformation theory for metal plasticity. It can be readily calibrated using standardized mechanical tests and implemented in finite element codes as a user-defined material model. In our experience with the use of the Genin-Hutchinson model in finite element calculations, we have found that, in some instances, the calculations suffer from numerical convergence problems. In the present study, we show that the problems stem from the fact that the model, in its present form, can become unstable in the post-matrix cracking regime, even when the stress-strain curves used for calibration exhibit strain hardening.

The objectives of the study are twofold. The first is to identify the source of instability and ascertain the role of the stress-strain relations used as calibrating inputs. The second is to remedy the model formulation to ensure stability for strain-hardening materials. The modifications to the original model are necessarily minimal, in order to retain the positive features of the original formulation: notably, the excellent agreement between experimental and predicted stress-strain curves for $45^{\circ}$ tension and pure shear [1], as well as between experimental and predicted strains in notched tension tests [2].

\footnotetext{
* Corresponding author. Tel.: +1 8058932694.

E-mail address: varun_rajan@umail.ucsb.edu (V.P. Rajan).
}

The outline of the paper is as follows. First, we recapitulate the essential features of the original GH model. Second, we derive a mathematically-equivalent incremental version of the model. Here the increments in stress are related to increments in strain via a tangential stiffness matrix that depends on the current stress state. Third, we demonstrate that, for certain anisotropic laminates, the incremental model, and hence the original model, can be unstable after matrix cracking. Fourth, we modify the incremental formulation to ensure stability. This is accomplished by judiciously adjusting the entries in the tangential stiffness matrix so it is positive-definite for all strain-hardening laminates. The remedy has been devised to not only ensure stability but also satisfy a number of additional conditions, notably, that: (i) the stress-strain relationships in the elastic domain are preserved exactly; (ii) the responses in $0^{\circ}$ tension, $45^{\circ}$ tension, equibiaxial tension, and pure shear are recovered; (iii) it reduces to the original model for the special case of a quasi-isotropic laminate; and (iv) it be based in part on the mechanics of CMC laminates after matrix cracking (rather than being purely mathematical). We show here that the modified version of the model satisfies all of the aforementioned conditions. Finally, we apply this model to simulate open-hole tension tests of CMC plates. Whereas the finite element simulations employing the original model fail to converge for certain inelastically-anisotropic laminates, the new model exhibits no such convergence problems. Furthermore, the original and modified models yield identical results for quasi-isotropic laminates, as required.

The weakness of the remediation approach is that it is necessarily ad hoc. Ideally, in converting a deformation theory into an 
incremental theory, a yield surface and a flow rule would be utilized. However, our ad hoc incremental formulation should be sufficient for scenarios in which material elements undergo nearly proportional straining: a limitation also of the original $\mathrm{GH}$ formulation.

\section{Original formulation of GH model}

In its original formulation, the $\mathrm{GH}$ model expresses the stress state in terms of the total strain components. The procedure, described in Genin and Hutchinson [1], is summarized below. It is restricted to loadings with proportional straining and thus the axes of principal strain are assumed not to rotate significantly during deformation.

The contributions of principal stresses to the principal strains are assumed to be additive when the principal strains are oriented along directions of material symmetry. For principal strains oriented at $0^{\circ}$, the strains are written as

$\epsilon_{I}=f_{0}\left(\sigma_{I}\right)+f_{0 T}\left(\sigma_{I I}\right)$

$\epsilon_{I I}=f_{0 T}\left(\sigma_{I}\right)+f_{0}\left(\sigma_{I I}\right)$

Similarly, for principal strains oriented at $45^{\circ}$ to the fiber directions:

$\epsilon_{I}=f_{45}\left(\sigma_{I}\right)+f_{45 T}\left(\sigma_{I I}\right)$

$\epsilon_{I I}=f_{45 T}\left(\sigma_{I}\right)+f_{45}\left(\sigma_{I I}\right)$

Here the functions $f$ are stress-strain curves measured in uniaxial tension tests conducted at either $0^{\circ}$ or $45^{\circ}$. $f_{0}$ and $f_{45}$ refer to normal strains aligned with the load axis, whereas $f_{0 T}$ and $f_{45 T}$ refer to normal strains transverse to the load axis. Considering the special case of equibiaxial loading $\left(\sigma_{I}=\sigma_{I I}=\sigma\right)$, wherein the axes of principal stress are indeterminate, the four functions $f$ are found to be related by

$f_{0}(\sigma)+f_{0 T}(\sigma)=f_{45}(\sigma)+f_{45 T}(\sigma)$

Hence, only three of the four functions are independent. Eq. (3) has been shown to be approximately satisfied by stress-strain curves measured on a $\mathrm{SiC} / \mathrm{CAS}\left[0^{\circ} / 90^{\circ}\right]$ laminate [1].

Non-linearity in the GH model is couched in terms of so-called 'stress deficits': that is, differences between elastic and actual stress values. The elastic stresses are obtained from Hooke's law. For instance, when the principal strains are oriented at $0^{\circ}$, the elastic (principal) stresses are given by

$$
\left[\begin{array}{c}
\sigma_{e l, I}^{0} \\
\sigma_{e l, I I}^{0}
\end{array}\right]=\left[\begin{array}{cc}
\frac{E_{0}}{1-v_{0}^{2}} & \frac{v_{0} E_{0}}{1-v_{0}^{2}} \\
\frac{v_{0} E_{0}}{1-v_{0}^{2}} & \frac{E_{0}}{1-v_{0}^{2}}
\end{array}\right]\left[\begin{array}{c}
\epsilon_{I} \\
\epsilon_{I I}
\end{array}\right]=\mathbf{C}_{\boldsymbol{e l}}^{\mathbf{0}}\left[\begin{array}{c}
\epsilon_{I} \\
\epsilon_{I I}
\end{array}\right]
$$

where $\boldsymbol{C}_{\boldsymbol{e l}}^{\mathbf{0}}$ is the elastic stiffness matrix for principal strains oriented at $0^{\circ}$.

Similarly, the elastic (principal) stresses for principal strains oriented at $45^{\circ}$ are

$$
\left[\begin{array}{c}
\sigma_{e l, I}^{45} \\
\sigma_{e l, I I}^{45}
\end{array}\right]=\left[\begin{array}{cc}
\frac{E_{45}}{1-v_{45}^{2}} & \frac{v_{45} E_{45}}{1-v_{45}^{2}} \\
\frac{v_{45} E_{45}}{1-v_{45}^{2}} & \frac{E_{45}}{1-v_{45}^{2}}
\end{array}\right]\left[\begin{array}{c}
\epsilon_{I} \\
\epsilon_{I I}
\end{array}\right]=\boldsymbol{C}_{\boldsymbol{e l}}^{\mathbf{4 5}}\left[\begin{array}{c}
\epsilon_{I} \\
\epsilon_{I I}
\end{array}\right]
$$

Using these results, the stress deficits at $0^{\circ}$ can be written as

$$
\left[\begin{array}{c}
\Delta \sigma_{I}^{0} \\
\Delta \sigma_{I I}^{0}
\end{array}\right]=\boldsymbol{C}_{\boldsymbol{e l}}^{\mathbf{0}}\left[\begin{array}{c}
\epsilon_{I} \\
\epsilon_{I I}
\end{array}\right]-\left[\begin{array}{c}
\Sigma^{0}\left(\epsilon_{I}, \epsilon_{I I}\right) \\
\Sigma^{0}\left(\epsilon_{I I}, \epsilon_{I}\right)
\end{array}\right]
$$

The $\Sigma$ terms in Eq. (6) correspond to the solution (inversion) of Eq. (1). A similar statement can be written for the $45^{\circ}$ direction, with the $\Sigma$ terms corresponding to the solution (inversion) of Eq. (2).

To obtain the stress deficits at the angle of principal strains, $\theta_{\epsilon}$, the stress deficits at $0^{\circ}$ and $45^{\circ}$ are interpolated in accordance with
$\Delta \sigma_{I}^{\theta_{\epsilon}}=\Delta \sigma_{I}^{0} \cos ^{2}\left(2 \theta_{\epsilon}\right)+\Delta \sigma_{I}^{45} \sin ^{2}\left(2 \theta_{\epsilon}\right)$

$\Delta \sigma_{I I}^{\theta_{\epsilon}}=\Delta \sigma_{I I}^{0} \cos ^{2}\left(2 \theta_{\epsilon}\right)+\Delta \sigma_{I I}^{45} \sin ^{2}\left(2 \theta_{\epsilon}\right)$

Combining the previous results, we find:

$$
\begin{array}{r}
{\left[\begin{array}{l}
\sigma_{I}^{\theta_{\epsilon}} \\
\sigma_{I I}^{\theta_{\epsilon}}
\end{array}\right]=\boldsymbol{C}_{\boldsymbol{e l}}^{\theta_{\epsilon}}\left[\begin{array}{c}
\epsilon_{I} \\
\epsilon_{I I}
\end{array}\right]-\cos ^{2}\left(2 \theta_{\epsilon}\right)\left(\boldsymbol{C}_{\boldsymbol{e l}}^{\mathbf{0}}\left[\begin{array}{c}
\epsilon_{I} \\
\epsilon_{I I}
\end{array}\right]-\left[\begin{array}{c}
\Sigma^{0}\left(\epsilon_{I}, \epsilon_{I I}\right) \\
\Sigma^{0}\left(\epsilon_{I I}, \epsilon_{I}\right)
\end{array}\right]\right)} \\
-\sin ^{2}\left(2 \theta_{\epsilon}\right)\left(\boldsymbol{C}_{\boldsymbol{e l}}^{\mathbf{4 5}}\left[\begin{array}{c}
\epsilon_{I} \\
\epsilon_{I I}
\end{array}\right]-\left[\begin{array}{c}
\Sigma^{45}\left(\epsilon_{I}, \epsilon_{I I}\right) \\
\Sigma^{45}\left(\epsilon_{I I}, \epsilon_{I}\right)
\end{array}\right]\right)
\end{array}
$$

It can further be shown through standard procedures of coordinate transformations that

$\boldsymbol{C}_{\boldsymbol{e l}}^{\boldsymbol{\theta}}=\boldsymbol{C}_{\boldsymbol{e l}}^{\mathbf{0}} \cos ^{2}(2 \theta)+\boldsymbol{C}_{\boldsymbol{e l}}^{\mathbf{4 5}} \sin ^{2}(2 \theta)$

so Eq. (8) reduces to

$\left[\begin{array}{c}\sigma_{I}^{\theta_{\epsilon}} \\ \sigma_{I I}^{\theta_{\epsilon}}\end{array}\right]=\cos ^{2}\left(2 \theta_{\epsilon}\right)\left[\begin{array}{c}\Sigma^{0}\left(\epsilon_{I}, \epsilon_{I I}\right) \\ \Sigma^{0}\left(\epsilon_{I I}, \epsilon_{I}\right)\end{array}\right]+\sin ^{2}\left(2 \theta_{\epsilon}\right)\left[\begin{array}{c}\Sigma^{45}\left(\epsilon_{I}, \epsilon_{I I}\right) \\ \Sigma^{45}\left(\epsilon_{I I}, \epsilon_{I}\right)\end{array}\right]$

Because the shear stress deficit is assumed to be zero, the actual shear stress (again, oriented at $\theta_{\epsilon}$ ) is merely the elastic shear stress:

$\tau^{\theta_{\epsilon}}=\frac{E_{45}-E_{0}}{2\left(1+v_{45}\right)\left(1+v_{0}\right)}\left(\epsilon_{I}-\epsilon_{I I}\right) \sin \left(4 \theta_{\epsilon}\right)$

\section{Incremental formulation of GH model}

We seek to convert the original GH model into an incremental formulation. Not only is the incremental formulation useful for assessing stability, but it is also amenable to modification to correct instabilities, as demonstrated below.

\subsection{Approach}

Consider two strain/stress states: the current state, denoted by the superscript $c$, and the new state, denoted by the superscript $n$. The states are related to one another by a prescribed strain increment. The goal is to determine the new stress state (or, equivalently, the increments in each of the stress components) in terms of the strain increment and the current strain and stress states.

The current strain state is given by two principal strains oriented at the principal angle, $\theta_{\epsilon}^{c}$, expressed generally as:

$\boldsymbol{\epsilon}^{\boldsymbol{c}}=\left[\epsilon_{I}^{c}, \epsilon_{I I}^{c}, 0\right]$

The increment in strain is also oriented at $\theta_{\epsilon}^{c}$. Although we assume proportional straining, we consider a non-zero shear strain component in order to assess stability with respect to any incremental deformation. The incremental strain tensor is thus expressed as:

$\mathbf{d} \epsilon=\left[\mathrm{d} \epsilon_{I}, \mathrm{~d} \epsilon_{I I}, \mathrm{~d} \gamma\right]$

Increments in principal strains are first-order in $\mathrm{d} \epsilon_{I}$ and $\mathrm{d} \epsilon_{I I}$, but are only second-order in $\mathrm{d} \gamma$. If the increment is small, the latter term is negligible. Conversely, the increment in $\theta_{\epsilon}$ (the orientation of the principal strain axes) is first-order in $\mathrm{d} \gamma$, and higher-order in the other components. Therefore, the shear strain increment rotates the principal axes while leaving the magnitudes of the principal strains unchanged, and vice versa for the normal strain increments.

The new strain state, oriented at an angle $\theta_{\epsilon}^{n}$, is thus given by

$\boldsymbol{\epsilon}^{\boldsymbol{n}}=\left[\epsilon_{I}^{o}+\mathrm{d} \epsilon_{I}, \epsilon_{I I}^{o}+\mathrm{d} \epsilon_{I I}, 0\right]$

where

$\theta_{\epsilon}^{n}=\theta_{\epsilon}^{c}+\mathrm{d} \theta_{\epsilon}$

and 
$\mathrm{d} \theta_{\epsilon}=\frac{\mathrm{d} \gamma}{2\left(\epsilon_{I}^{o}-\epsilon_{I I}^{o}\right)}$

The stresses in the current state are also rotated into $\theta_{\epsilon}^{c}$. Since the principal axes of stress and strain do not necessarily coincide, a nonzero shear stress component may also be present, and thus the current stress state is written as

$\boldsymbol{\sigma}^{c}=\left[\sigma_{I}^{c}, \sigma_{I I}^{c}, \tau^{c}\right]$

\subsection{Formulation}

We derive the incremental model by linearizing Eqs. (10) and (11) about $\mathbf{d} \epsilon=0$.

Using Eq. (1), we obtain

$\left[\begin{array}{c}\mathrm{d} \epsilon_{I} \\ \mathrm{~d} \epsilon_{I I}\end{array}\right]=\left[\begin{array}{cc}f_{0}^{\prime}\left(\sigma_{I}^{0}\right) & f_{0 T}^{\prime}\left(\sigma_{I I}^{0}\right) \\ f_{0 T}^{\prime}\left(\sigma_{I}^{0}\right) & f_{0}^{\prime}\left(\sigma_{I I}^{0}\right)\end{array}\right]\left[\begin{array}{l}\mathrm{d} \sigma_{I}^{0} \\ \mathrm{~d} \sigma_{I I}^{0}\end{array}\right]=\boldsymbol{S}^{\mathbf{0}}(\boldsymbol{\sigma})\left[\begin{array}{c}\mathrm{d} \sigma_{I}^{0} \\ \mathrm{~d} \sigma_{I I}^{0}\end{array}\right]$

where the prime symbols denote derivatives. Similarly, using Eq. (2):

$$
\left[\begin{array}{c}
\mathrm{d} \epsilon_{I} \\
\mathrm{~d} \epsilon_{I I}
\end{array}\right]=\left[\begin{array}{ll}
f_{45}^{\prime}\left(\sigma_{I}^{45}\right) & f_{45 T}^{\prime}\left(\sigma_{I I}^{45}\right) \\
f_{45 T}^{\prime}\left(\sigma_{I}^{45}\right) & f_{45}^{\prime}\left(\sigma_{I I}^{45}\right)
\end{array}\right]\left[\begin{array}{l}
\mathrm{d} \sigma_{I}^{45} \\
\mathrm{~d} \sigma_{I I}^{45}
\end{array}\right]=\boldsymbol{S}^{\mathbf{4 5}}(\boldsymbol{\sigma})\left[\begin{array}{l}
\mathrm{d} \sigma_{I}^{45} \\
\mathrm{~d} \sigma_{I I}^{45}
\end{array}\right]
$$

Expanding Eq. (10) about $\mathbf{d} \epsilon=0$ yields

$$
\begin{aligned}
{\left[\begin{array}{c}
\sigma_{I}^{n} \\
\sigma_{I I}^{n}
\end{array}\right] } & =\cos ^{2}\left(2\left(\theta_{\epsilon}^{c}+\mathrm{d} \theta_{\epsilon}\right)\right)\left[\begin{array}{l}
\Sigma^{0}\left(\epsilon_{I}^{c}+\mathrm{d} \epsilon_{I}, \epsilon_{I I}^{c}+\mathrm{d} \epsilon_{I I}\right) \\
\Sigma^{0}\left(\epsilon_{I I}^{c}+\mathrm{d} \epsilon_{I I}, \epsilon_{I}^{c}+\mathrm{d} \epsilon_{I}\right)
\end{array}\right] \\
& +\sin ^{2}\left(2\left(\theta_{\epsilon}^{c}+\mathrm{d} \theta_{\epsilon}\right)\right)\left[\begin{array}{l}
\Sigma^{45}\left(\epsilon_{I}^{c}+\mathrm{d} \epsilon_{I}, \epsilon_{I I}^{c}+\mathrm{d} \epsilon_{I I}\right) \\
\Sigma^{45}\left(\epsilon_{I I}^{c}+\mathrm{d} \epsilon_{I I}, \epsilon_{I}^{c}+\mathrm{d} \epsilon_{I}\right)
\end{array}\right]
\end{aligned}
$$

where $\sigma_{I, I}^{n}$ are normal stresses oriented at $\theta_{\epsilon}^{n}$. Substituting the previous results into Eq. (20) and neglecting higher-order terms yields

$$
\begin{aligned}
& {\left[\begin{array}{l}
\mathrm{d} \sigma_{I} \\
\mathrm{~d} \sigma_{I I}
\end{array}\right]=2 \sin \left(4 \theta_{\epsilon}^{c}\right) \mathrm{d} \theta_{\epsilon}\left(\left[\begin{array}{c}
\Sigma^{45}\left(\epsilon_{I}^{c}, \epsilon_{I I}^{c}\right) \\
\Sigma^{45}\left(\epsilon_{I I}^{c}, \epsilon_{I}^{c}\right)
\end{array}\right]-\left[\begin{array}{c}
\Sigma^{0}\left(\epsilon_{I}^{c}, \epsilon_{I}^{c}\right) \\
\Sigma^{0}\left(\epsilon_{I I}^{c}, \epsilon_{I}^{c}\right)
\end{array}\right]\right)} \\
& +\left(\left[\boldsymbol{S}^{\mathbf{0}}\left(\boldsymbol{\sigma}^{\boldsymbol{c}}\right)\right]^{-1} \cos ^{2}\left(2 \theta_{\epsilon}^{c}\right)+\left[\boldsymbol{S}^{\mathbf{4 5}}\left(\boldsymbol{\sigma}^{\boldsymbol{c}}\right)\right]^{-1} \sin ^{2}\left(2 \theta_{\epsilon}^{c}\right)\right)\left[\begin{array}{l}
\mathrm{d} \epsilon_{I} \\
\mathrm{~d} \epsilon_{I I}
\end{array}\right]
\end{aligned}
$$

Note that the first term scales with the product of a modulus and $\mathrm{d} \gamma$ (per Eq. (16)), whereas the second term scales with the product of a modulus and $\mathrm{d} \epsilon_{I, I I}$. For nearly proportional straining, the former term is negligible in comparison to the latter. So, we obtain:

$$
\left[\begin{array}{l}
\mathrm{d} \sigma_{I} \\
\mathrm{~d} \sigma_{I I}
\end{array}\right]=\left(\left[\boldsymbol{S}^{\mathbf{0}}\left(\boldsymbol{\sigma}^{\boldsymbol{c}}\right)\right]^{-1} \cos ^{2}\left(2 \theta_{\epsilon}^{c}\right)+\left[\boldsymbol{S}^{\mathbf{4 5}}\left(\boldsymbol{\sigma}^{\boldsymbol{c}}\right)\right]^{-1} \sin ^{2}\left(2 \theta_{\epsilon}^{c}\right)\right)\left[\begin{array}{l}
\mathrm{d} \epsilon_{I} \\
\mathrm{~d} \epsilon_{I I}
\end{array}\right]
$$

Finally, linearizing the shear stress equation yields

$$
\begin{aligned}
\mathrm{d} \tau= & \frac{E_{45}-E_{0}}{2\left(1+v_{45}\right)\left(1+v_{0}\right)} \\
& \times\left(4\left(\epsilon_{I}^{c}-\epsilon_{I I}^{c}\right) \cos \left(4 \theta_{\epsilon}^{c}\right) \mathrm{d} \theta_{\epsilon}+\left(\mathrm{d} \epsilon_{I}-\mathrm{d} \epsilon_{I I}\right) \sin \left(4 \theta_{\epsilon}^{c}\right)\right)
\end{aligned}
$$

\section{Assessment of stability}

\subsection{Stability criterion}

As discussed by Hill [3], a structure is stable if the condition

$\mathrm{d}^{2} W=\int_{V} \frac{1}{2} \mathbf{d} \boldsymbol{\sigma}: \mathbf{d} \epsilon \mathrm{d} V \geqslant 0$

is satisfied for the strain increment $\mathbf{d} \boldsymbol{\epsilon}$ associated with any incremental displacement dq of the structure [4]. For Eq. (24) to hold, it is sufficient that the second-order work be positive 'in the small' $[5,6]$. This implies that the condition

$$
\mathbf{d} \boldsymbol{\sigma}: \mathbf{d} \epsilon \geqslant 0
$$

should be satisfied locally, for every material element. Eq. (25) imposes a condition on the constitutive law: notably, that the tangential stiffness matrix, $C_{i j}=\mathrm{d} \sigma_{i} / \mathrm{d} \epsilon_{j}$, be positive-definite [6], or, equivalently, that the symmetric part of the tangential stiffness, $\left(\boldsymbol{C}+\boldsymbol{C}^{\boldsymbol{T}}\right) / 2$, possess no negative eigenvalues. This condition will be used to assess stability in the present work. Since Eq. (25) implies positive material stiffness in every strain direction, it is not expected to hold for strain states in which the input stress-strain curves exhibit strain-softening. Conversely, the stability condition should be satisfied when the input stress-strain curves exhibit strain-hardening.

The incremental formulation, described above, involves changes in the magnitudes of the stress components and rotation of the stress vector through the angle $\mathrm{d} \theta_{\epsilon}$. To assess stability using Eq. (25), the incremental stress and strain vectors must be written with respect to the same coordinate axes. For this purpose, we rotate the new stress vector, oriented at $\theta_{\epsilon}^{n}$, back to the current principal axes, oriented at $\theta_{\epsilon}^{c}$. Neglecting higher-order terms, the principal stress increment (Eq. (22)) remains unchanged, but the shear stress increment has an additional term, now written as:

$$
\begin{aligned}
\mathrm{d} \tau^{c}= & \frac{E_{45}-E_{0}}{2\left(1+v_{45}\right)\left(1+v_{0}\right)} \\
& \times\left(4\left(\epsilon_{I}^{c}-\epsilon_{I I}^{c}\right) \cos \left(4 \theta_{\epsilon}^{c}\right) \mathrm{d} \theta_{\epsilon}+\left(\mathrm{d} \epsilon_{I}-\mathrm{d} \epsilon_{I I}\right) \sin \left(4 \theta_{\epsilon}^{c}\right)\right) \\
& +\left(\sigma_{I}^{c}-\sigma_{I I}^{c}\right) \mathrm{d} \theta_{\epsilon}
\end{aligned}
$$

Using Eqs. (22) and (26), we can now assess the stability of the incremental formulation. Furthermore, since the two formulations of the model are mathematically equivalent, conclusions regarding the stability of the incremental formulation are also applicable to the original formulation.

Using the preceding framework, we find that two types of instabilities can be obtained in the inelastic regime: one associated with Eq. (26), and the other with Eq. (22). Each, in turn, is described below.

\subsection{Shear strain instability}

Establishing general conditions for stability is difficult (and not particularly enlightening), so a special case is examined here. We consider a uniaxial tension test conducted in the $0^{\circ}$ direction. The material is assumed to have $v_{0}=0$ (not unreasonable for $\mathrm{CMCs}$ ). Then, we consider an incremental shear strain. Obviously, $\epsilon_{I I}^{c}=0, \sigma_{I I}^{c}=0, \mathrm{~d} \epsilon_{I}=\mathrm{d} \epsilon_{I I}=0, \theta_{\epsilon}^{c}=0$ and $v_{45}=1-E_{45} / E_{0}$. Defining $\beta=E_{45} / E_{0}$, Eq. (26) simplifies to

$\mathrm{d} \tau^{c}=\frac{-2 E_{0}(1-\beta)}{(2-\beta)} \epsilon_{I}^{c} \mathrm{~d} \theta_{\epsilon}+\sigma_{I}^{c} \mathrm{~d} \theta_{\epsilon}$

A good approximation of the first principal stress is $\sigma_{I}^{c}=\alpha E_{0}\left(\epsilon_{I}^{c}-\epsilon_{m c}\right)+E_{0} \epsilon_{m c}$, where $\alpha$ is the ratio of the post-matrix cracking modulus to the pre-matrix cracking modulus in the $0^{\circ}$ direction, and $\epsilon_{m c}$ is the matrix cracking strain. Since $\mathrm{d} \theta_{\epsilon}$ is proportional to $\mathrm{d} \gamma$, stability requires that $\mathrm{d} \tau^{c} \mathrm{~d} \theta_{\epsilon} \geqslant 0$. Following some algebra, we obtain the stability condition:

$$
1-\alpha-\frac{1}{\epsilon_{I}^{c} / \epsilon_{m c}-1} \leqslant \frac{\beta}{2-\beta}
$$

It is trivial to show that, although this equation is automatically satisfied for elastically-isotropic materials $(\beta=1)$, it is not generally true for elastically-anisotropic materials. For one set of typical values of material parameters $\left(\alpha=1 / 4, \epsilon_{m c}=0.001, \epsilon_{I}^{c}=0.006\right)$, stability requires that $\beta$ exceed 0.71 . Laminates in which the fiber modulus is significantly higher than the matrix modulus (perhaps 
because of remnant porosity in the matrix) are unlikely to satisfy this condition.

\subsection{Principal strain instability}

Here, we consider the case of a uniaxial tension test conducted at $45^{\circ}$. In this case, Eq. (22) reduces to

$$
\left[\begin{array}{l}
\mathrm{d} \sigma_{I}^{c} \\
\mathrm{~d} \sigma_{I I}^{c}
\end{array}\right]=\left[\boldsymbol{S}^{\mathbf{4 5}}\left(\boldsymbol{\sigma}^{\boldsymbol{c}}\right)\right]^{-1}\left[\begin{array}{l}
\mathrm{d} \epsilon_{I} \\
\mathrm{~d} \epsilon_{I I}
\end{array}\right]
$$

and thus the stability condition is satisfied if $\boldsymbol{S}^{\mathbf{4 5}}$ is positive-definite. Note that, after matrix cracking, the compliances in the $45^{\circ}$ and $45^{\circ}$ transverse directions are essentially equal and of opposite sign because of 'fiber scissoring' (see Eq. (3)). So, we obtain

$$
\boldsymbol{S}^{\mathbf{4 5}}=\left[\begin{array}{cc}
f_{45}^{\prime}\left(\sigma_{I}^{45}\right) & f_{45 T}^{\prime}\left(\sigma_{I I}^{45}\right) \\
f_{45 T}^{\prime}\left(\sigma_{I}^{45}\right) & f_{45}^{\prime}\left(\sigma_{I I}^{45}\right)
\end{array}\right]=\left[\begin{array}{cc}
1 / E_{45, p m c} & -v_{45} / E_{45} \\
-1 / E_{45, p m c} & 1 / E_{45}
\end{array}\right]
$$

where the subscript 'pmc' denotes post-matrix cracking. We now consider a material that is elastically-isotropic, but inelastically very anisotropic. For instance, taking $E_{0}=E_{45}=200 \mathrm{GPa}, v_{0}=$ $v_{45}=0.1$, and $E_{45 \text {,pmc }}=5 \mathrm{GPa}$, it is readily seen that $\boldsymbol{S}^{45}$ is non-positive-definite. This result arises because of the large dissimilarity in the off-diagonal terms that describe coupling between strains and stresses in orthogonal directions.

\subsection{Numerical calculations}

To illustrate the effects of the instabilities in finite element analyses, the uniaxial tension tests described above were simulated using ABAQUS Standard (Version 6-9.2, Dassault Systèmes) with the original GH model as a user-defined constitutive law. Relatively coarse meshes (16 four-noded, quadrilateral, plane stress elements), depicted in Fig. $1 \mathrm{~b}$ and d, were used. (Note that mesh refinement does not fix, and can actually exacerbate, the convergence problems associated with unstable materials.) In an attempt to suppress local material instabilities, automatic stabilization (damping) was employed.

The input stress-strain curves for the $0^{\circ}$ tension test of the elastically-anisotropic material are shown in Fig. 1a. The stress-strain curves are bi-linear, with elastic constants $E_{0}=200 \mathrm{GPa}, v_{0}=0.1$, and $E_{45}=100 \mathrm{GPa}$. Thus $\beta=E_{45} / E_{0}=0.5$, below the predicted critical value for stability (0.71) in Eq. (28). In the post-matrix cracking regime, the tangent moduli of the $0^{\circ}$ and $45^{\circ}$ stress-strain curves are equal.

The stress in the direction of loading is plotted in Fig. 2a for all integration points within the mesh. In the elastic regime and in a portion of the inelastic regime, each element possesses a positive-definite tangential stiffness matrix. No instability results, and the stress-strain curve predicted by the finite element analysis therefore agrees with the $0^{\circ}$ stress-strain curve $\left(f_{0}\right)$ used as input. However, at a sufficiently large strain in the inelastic regime, the smallest eigenvalue of the symmetric part of the tangential stiffness matrix becomes negative (see Fig. 2c), causing the stiffness matrix to lose positive-definiteness. When this occurs, the numerical solver (based on the Newton-Raphson method) encounters convergence difficulties. As a consequence, the stresses at the integration points diverge from the $0^{\circ}$ stress-strain curve. The magnitude of the errors exceeds $25 \%$.

Next we consider the tensile response of an inelastically-anisotropic material (defined in Fig. 1c) in the $45^{\circ}$ orientation. Once again, bi-linear behavior is assumed. The material is elasticallyisotropic, so the shear strain-induced instability illustrated above does not arise. However, inelastic anisotropy is present: the ratio of the post-matrix cracking moduli being $E_{45, p m c} / E_{0, p m c}=1 / 100$.
The normal stress in the $0^{\circ}$ direction (not in the loading direction) is plotted in Fig. $2 \mathrm{~b}$ for all integration points within the mesh. Once again, the elastic behavior is correctly modeled by the finite element simulation, while the inelastic behavior is poorly modeled due to convergence problems. Loss of positive-definiteness occurs immediately after matrix cracking, as indicated in Fig. 2d. The errors in the computed stresses exceed $20 \%$ for several integration points. (The normal stress in the loading direction - at $45^{\circ}$ to the fiber axes - displays lower error, because errors of opposite sign in the normal and shear stresses at $0^{\circ}$ tend to cancel one another out.)

In the relatively simple loadings and geometries considered here, the finite element analyses yield inaccurate results. In other circumstances (such as those considered below), the finite element solver can fail to converge entirely. This can occur, for instance, when more complex geometries are simulated.

\section{Remediation of instabilities}

As demonstrated, the instabilities associated with the original model prevent accurate finite element simulations for certain anisotropic materials. The problem is remedied by adjusting the entries of the tangential stiffness matrix in the inelastic domain. The adjustments are physically motivated, as opposed to being purely mathematical. One adjustment corrects for the problem of the shear strain instability in elastically-anisotropic materials; the other addresses the problem of the principal strain instability in inelastically-anisotropic materials. Stability of the new model is ensured by verifying that the tangential stiffness matrix is positive-definite when the input stress-strain curves exhibit strainhardening.

\subsection{Shear strain instability}

Prior to matrix cracking, shear-extension coupling can exist if $E_{0} \neq E_{45}$. Specifically, a difference in principal strains causes shear stresses to develop if the orientation of the principal axes lies between $0^{\circ}$ and $45^{\circ}$. The original $\mathrm{GH}$ model predicts that, even after matrix cracking, shear stresses within the material continue to rise with additional normal strain. This scenario is somewhat implausible, however, since matrix cracking alleviates the constraint that gives rise to shear-extension coupling. We expect, contrary to the original model, that matrix cracking causes a de-coupling of shear and extension, and thus no additional shear stress builds up from this coupling. We therefore assume that increments in shear stress in the inelastic regime are caused solely by increments in shear strain. But the proportionality constant cannot be chosen arbitrarily: the requirement of objectivity must be enforced [7]. Specifically, for quasi-isotropic materials, the axes of principal stress and strain must be aligned. Therefore, in the incremental model, these axes must rotate by the same angle. As noted by Bažant [7], this requirement is equivalent to the relationship

$\mathrm{d} \tau^{c}=\left(\sigma_{I}^{c}-\sigma_{I I}^{c}\right) \mathrm{d} \theta_{\epsilon}=\frac{\sigma_{I}^{c}-\sigma_{I I}^{c}}{2\left(\epsilon_{I}^{c}-\epsilon_{I I}^{c}\right)} \mathrm{d} \gamma$

where both the stress and the stress increment are oriented at $\theta_{\epsilon}^{c}$. Since the proportionality constant is guaranteed to be positive, this modification resolves the problem of shear strain instability. Note that Eq. (31) is identical to that in the original GH model for laminates that are elastically-isotropic $\left(E_{0}=E_{45}\right)$.

The elimination of the shear-extension coupling term renders the tangential stiffness matrix in the modified model orthotropic in the inelastic domain. Therefore, the modified model can be classified as an incrementally orthotropic model, as discussed by Bažant [7]. 


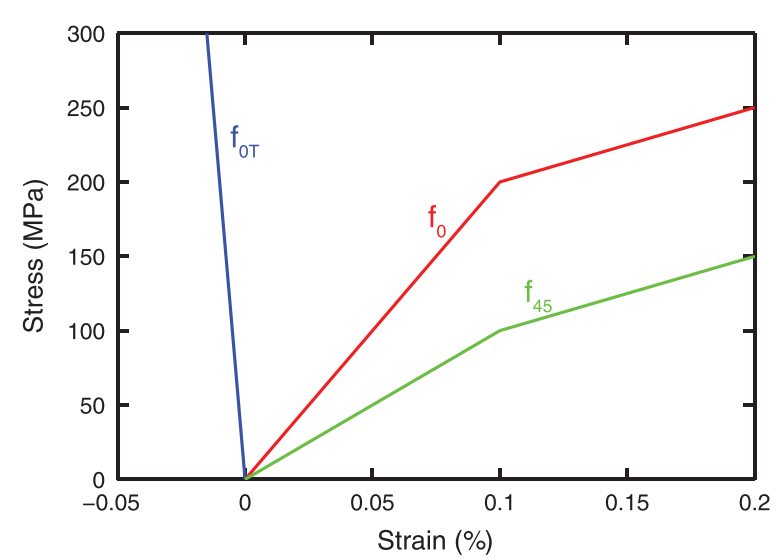

(a)

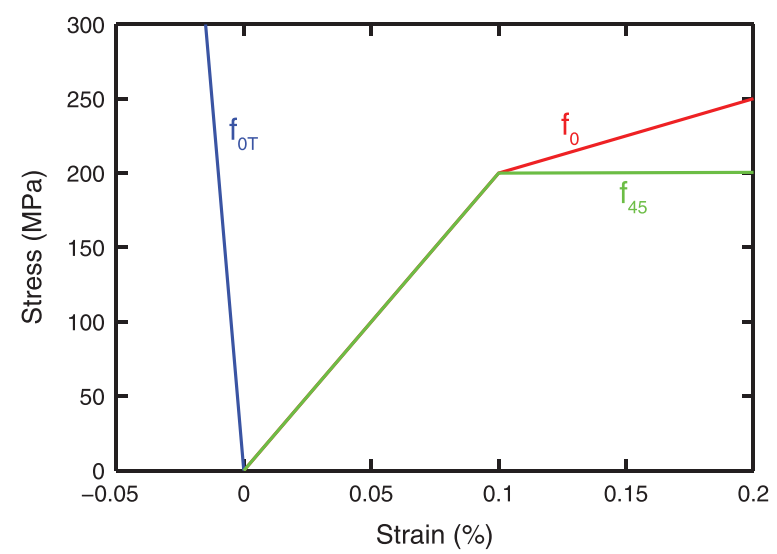

(c)

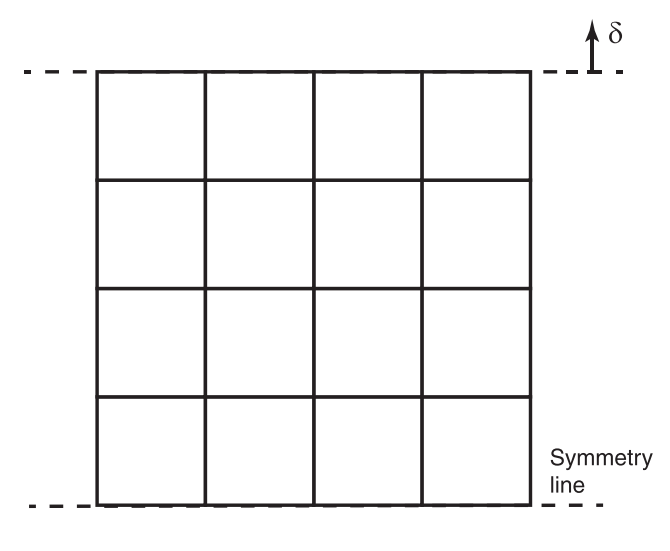

(b)

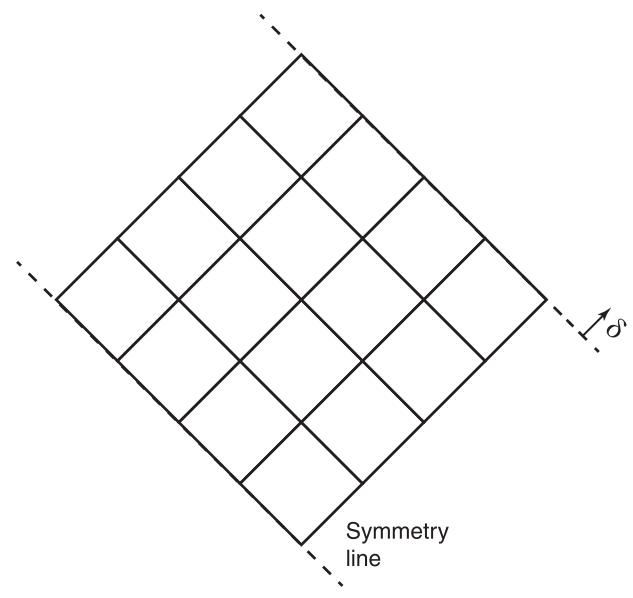

(d)

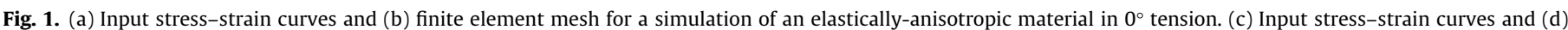

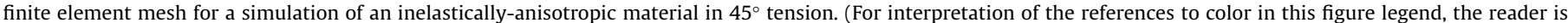
referred to the web version of this article.)

\subsection{Principal strain instability}

As noted previously, the principal strain instability arises because the description of the coupling between stresses and strains in the two principal directions is incomplete. To properly describe this coupling, the phenomenon of fiber scissoring must be taken into account.

Scissoring represents a low-stiffness deformation mode in which fibers rotate and bend in order to align themselves with the load axis. It occurs in the inelastic domain, wherein matrix cracks partially alleviate the constraints on fiber motion. Scissoring does not operate in the elastic domain (provided the matrix is sufficiently stiff) nor when the load is aligned with one of the fiber axes (i.e. at $0^{\circ}$ or $90^{\circ}$ ).

The GH model evidently fails to capture the scissoring mechanism. A particular example, shown in Fig. 5, illustrates the point. We consider a $\left[0^{\circ} / 90^{\circ}\right]$ laminate that is first loaded in tension at $45^{\circ}$ to the fiber axes, along the $I$-direction defined in Fig. 5. This loading produces a series of periodic matrix cracks normal to the $I$-direction. The laminate is subsequently loaded by a pair of incremental stresses $\Delta \sigma$ in the I- and II-directions, with $\Delta \sigma_{I I} \gg \Delta \sigma_{I}$. The compliance matrix from the original GH model, notably

$\boldsymbol{S}^{\mathbf{4 5}}=\left[\begin{array}{cc}f_{45}^{\prime}\left(\sigma_{I}^{45}\right) & f_{45 T}^{\prime}\left(\sigma_{I I}^{45}\right) \\ f_{45 T}^{\prime}\left(\sigma_{I}^{45}\right) & f_{45}^{\prime}\left(\sigma_{I I}^{45}\right)\end{array}\right]$ would predict that the incremental strain in the I-direction would be much greater than that in the II-direction. In contrast, upon consideration of the scissoring process, we conclude that, in fact, the magnitudes of the incremental strains would be reversed.

Here we propose an alternative compliance matrix to describe the strains resulting from fiber scissoring. To this end, the cracked matrix is conceptualized, to a first approximation, as a very compliant elastic medium with a large failure strain: an assumption also made in models of the post-cracking response of CMCs under $0^{\circ}$ loading [8]. In order for scissoring to be treated as an elastic phenomenon, the compliance matrix must be symmetric. (This symmetry makes sense in physical terms: when fiber scissoring is the predominant mode of deformation, large transverse strains should develop for stresses applied in either of the principal directions.) Additionally, the compliance matrix must preserve the $45^{\circ}$, $45^{\circ}$-transverse, and equibiaxial stress-strain relations. The simplest compliance matrix that satisfies these conditions is

$\boldsymbol{S}^{\mathbf{4 5}}=\left[\begin{array}{ll}f_{45}^{\prime}\left(\sigma_{I}^{45}\right) & f_{45 T}^{\prime}\left(\sigma_{I}^{45}\right) \\ f_{45 T}^{\prime}\left(\sigma_{I}^{45}\right) & f_{45}^{\prime}\left(\sigma_{I}^{45}\right)\end{array}\right]$

Here the second principal stress plays no role because the first principal stress governs the state of damage and therefore the propensity for scissoring to occur.

The response of real laminates is expected to fall between the extremes of Eq. (33), which accounts for fiber scissoring, and Eq. 

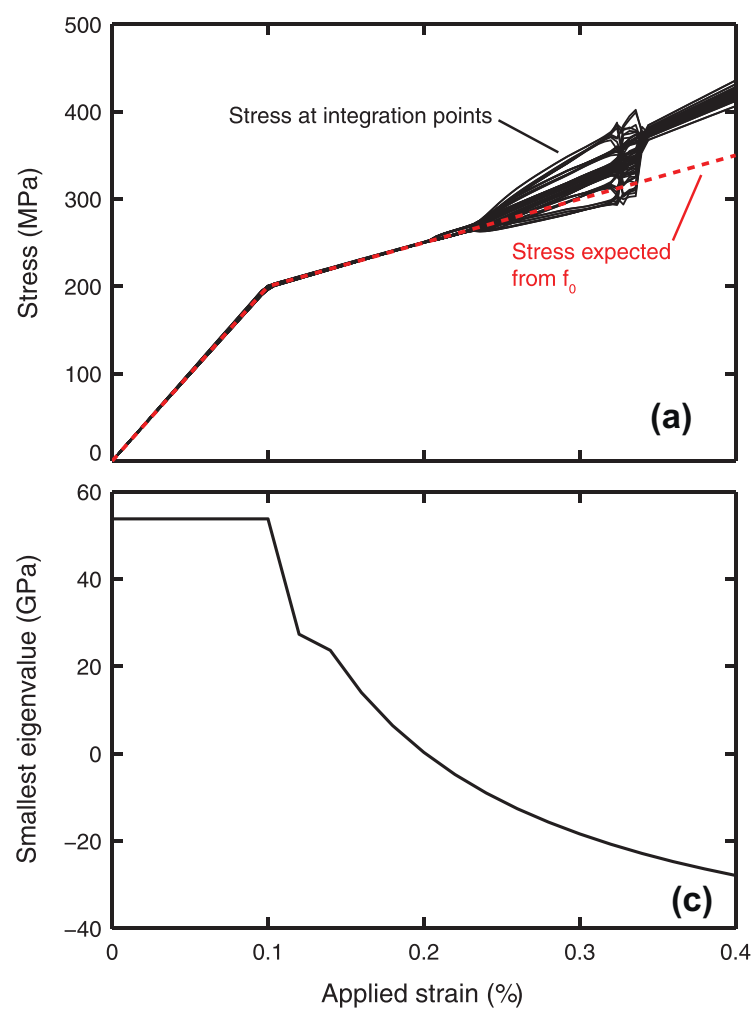
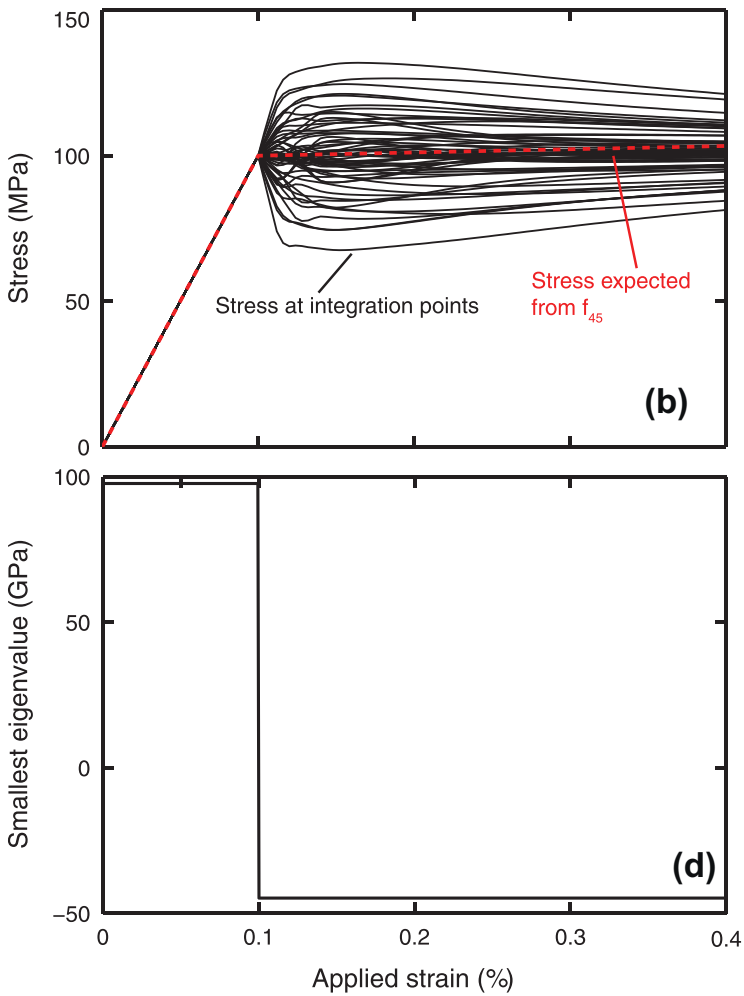

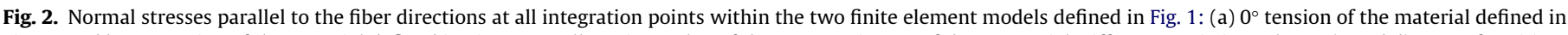

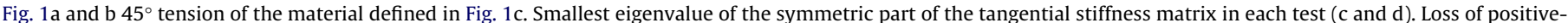

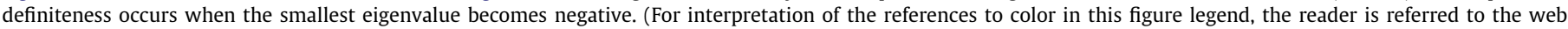
version of this article.)

(32), which accounts for fiber stretching and fragmentation. We assume that, for general loading in the $\theta$-direction, the pertinent compliance is a weighted sum of those in Eqs. (32) and (33). Enforcing the condition that the stress-strain curves for uniaxial tension at $0^{\circ}$ and $45^{\circ}$ should be recovered, the new compliance matrix becomes

$$
\boldsymbol{S}^{\theta}=D_{\theta}\left[\begin{array}{cc}
f_{\theta}^{\prime}\left(\sigma_{I}^{\theta}\right) & f_{\theta T}^{\prime}\left(\sigma_{I}^{\theta}\right) \\
f_{\theta T}^{\prime}\left(\sigma_{I}^{\theta}\right) & f_{\theta}^{\prime}\left(\sigma_{I}^{\theta}\right)
\end{array}\right]+\left(1-D_{\theta}\right)\left[\begin{array}{cc}
f_{\theta}^{\prime}\left(\sigma_{I}^{\theta}\right) & f_{\theta T}^{\prime}\left(\sigma_{I I}^{\theta}\right) \\
f_{\theta T}^{\prime}\left(\sigma_{I}^{\theta}\right) & f_{\theta}^{\prime}\left(\sigma_{I I}^{\theta}\right)
\end{array}\right]
$$

where $D_{\theta}$ is a constant that characterizes the tendency of the laminate to scissor in the $\theta$-direction. It is defined such that, when $D_{\theta}=1$, the compliance matrix is governed by fiber rotation (scissoring); conversely, when $D_{\theta}=0$, it is governed by fiber stretching and fragmentation.

In implementing this modification in the GH model, only $D_{0}$ and $D_{45}$ are required; in general, these quantities will be different from one another. In the $0^{\circ}$ direction, the fibers are aligned with the load axis, so there is no tendency to scissor and thus $D_{0}=0$. In contrast, in the $45^{\circ}$ direction, the laminate may undergo scissoring, so $0 \leqslant D_{45} \leqslant 1$. For a quasi-isotropic laminate, $D_{45}$ must equal 0 , since the $0^{\circ}$ and $45^{\circ}$ directions are equivalent. In contrast, for a $\left[0^{\circ} / 90^{\circ}\right]$ laminate, deformation is expected to be dominated by the scissoring mechanism; therefore, $D_{45}$ should be close to unity. Determination of the precise value of $D_{45}$ for the latter laminate is outside the scope of this work; micro-mechanical models may be required for this task. However, as shown in Section 6.2, stress distributions that arise during on-axis loading of $\left[0^{\circ} / 90^{\circ}\right]$ laminates are insensitive to $D_{45}$.

For $D_{45}=1$, the modified compliance matrix, and therefore the tangential stiffness matrix, is positive-definite when $f_{45}^{\prime}\left(\sigma_{I}^{45}\right)>$ $-f_{45 T}^{\prime}\left(\sigma_{I}^{45}\right)$. This condition is automatically satisfied, per Eq. (3).

\subsection{Material instability}

The aforementioned approach remedies material instability in the GH constitutive model. It yields a tangential stiffness matrix that is guaranteed to be positive-definite provided the stressstrain curves used for calibration exhibit strain hardening. One possible criticism of this strategy is that physically realistic instabilities, resulting from fiber fragmentation or shear banding $[9,10]$, may be artificially suppressed. The counter-argument is that the input stress-strain curves $f_{0}$ and $f_{45}$ could be readily modified to account for phenomena that lead to instabilities in the $0^{\circ}$ and $45^{\circ}$ directions. If, for instance, a strain softening portion were included in $f_{45}$, the model would predict a negative shear stiffness and the formation of a shear band; if, on the other hand, a softening portion were included in $f_{0}$, the model would predict a negative tensile stiffness and the formation of a tensile crack. Furthermore, since the $0^{\circ}$ and $45^{\circ}$ tension tests probe the extremes of the composite response, it seems unlikely that instabilities would occur for loadings at intermediate angles if these instabilities were not present in the input stress-strain curves. Although this approach requires further experimental and theoretical study for validation, it appears to offer a promising route for modelling material instability in CMC laminates.

\subsection{Criterion for inelasticity}

The modified constitutive model requires a criterion to distinguish between the elastic and inelastic regimes, since the procedures for computing stresses differ in the two regimes. For instance, in the original GH model, inelasticity is deemed to occur if any of the stress deficits are non-zero. But, this criterion leads to contradictory results if the matrix cracking stresses or strains differ 
in the $0^{\circ}$ and $45^{\circ}$ directions. To demonstrate the contradiction, we consider a material element undergoing equibiaxial stressing with a stress $\sigma=\sigma_{I}=\sigma_{I I}$ in the range $\sigma_{m c}^{45}<\sigma<\sigma_{m c}^{0}$. The original GH model predicts a nonzero stress deficit in the $45^{\circ}$ direction (since $\Delta \sigma_{I}^{45}>0$ ) and a zero stress deficit in the $0^{\circ}$ direction (since $\Delta \sigma_{I}^{0}=0$ ). Therefore, per Eq. (7), if we consider the principal stresses to be aligned at $45^{\circ}$, the material element is deemed to be inelastic, but if we consider the principal stresses to be aligned at $0^{\circ}$, the material element is deemed to be elastic. This result is contradictory since the axes of principal stress are arbitrary for equibiaxial stressing.

The contradiction arises from the assumption that matrix cracking is governed only by the largest principal stress: a natural consequence of the stress deficit criterion for matrix cracking. Therefore, if this criterion is used, both the original and modified GH models are appropriate only for materials that possess (nearly) identical matrix cracking stresses and strains in the $0^{\circ}$ and $45^{\circ}$ directions. While this condition may be approximately satisfied in CMCs with relatively dense, stiff matrices, e.g. SiC/CAS, it will not be satisfied in CMCs with compliant, weak matrices, e.g. C/C. (According to the categorization scheme of Evans and co-workers, the former composites are Class II materials whereas the latter are Class III. The classes can be distinguished by the ratio of the matrix shear modulus to the fiber Young's modulus [10].)

The modified GH model would be suitable for elastically-anisotropic materials provided that a different criterion for matrix cracking were employed. This criterion remains to be developed. As argued above, it would need to involve the second principal stress, to avoid the contradiction that arises for equibiaxial stressing.

In this work, the stress deficit criterion is used to assess the presence of matrix cracking within an element. Therefore, the finite element simulations that use the modified GH model are limited to elastically-isotropic laminates. For these materials, the shear strain instability discussed previously becomes irrelevant. However, the original GH model remains unsuitable for finite element calculations because of the principal strain instability that arises in inelastically-anisotropic materials.

\subsection{Predictions for simple loading scenarios}

By inspection, we see that the modified model agrees with the original model for $0^{\circ}$ and $45^{\circ}$ tension. The models also agree for equibiaxial tension, according to Eq. (3).

For the case of pure shear, the predictions of the original and modified models are somewhat different. In the original model, the tangent shear compliance in the inelastic domain is well approximated by

$\frac{\mathrm{d} \gamma}{\mathrm{d} \tau} \approx f_{45}^{\prime}(\tau)-f_{45 T}^{\prime}(\tau)$

In the modified model, the material is somewhat more compliant in shear:

$$
\frac{\mathrm{d} \gamma}{\mathrm{d} \tau} \approx\left(1+D_{45}\right)\left(f_{45}^{\prime}(\tau)-f_{45 T}^{\prime}(\tau)\right)
$$

Since the tangent modulus in the inelastic regime is usually very small (roughly $1-5 \mathrm{GPa}$ ) - that is, the response is nearly perfectlyplastic $[1,2]$ - the factor $1+D_{45}$ will have only a small influence on the shear flow stress. The predictions of the modified model are therefore expected to be almost indistinguishable from the experimental results for the loading scenarios considered in Genin and Hutchinson [1]: $0^{\circ}$ tension, $45^{\circ}$ tension, and pure shear.

For a quasi-isotropic laminate, the shear-extension coupling term is zero, and the material does not scissor in either the $0^{\circ}$ or $45^{\circ}$ direction, so $D_{0}=D_{45}=0$. Therefore, not only is the pure shear behavior identical in the modified and original models, but the models are actually equivalent. This fact is numerically confirmed in Section 6.

\section{Illustrative numerical examples}

\subsection{Simulations}

For ease of implementation, the constitutive equations in the modified model are integrated explicitly using the modified Euler scheme discussed by Sloan et al. [11]. The scheme utilizes automatic sub-stepping to limit the error arising from the integration procedure to within a prescribed error tolerance for each time step. It has been implemented in a user-material subroutine (UMAT) for use in ABAQUS.

The UMATs for the modified and original models are utilized to simulate an open-hole tension test of a composite laminate. Two materials are considered. In one, the laminate is elastically isotropic but inelastically anisotropic, with stress-strain curves identical to those depicted in Fig. 1c. In this simulation, the original $\mathrm{GH}$ model is expected to fail to converge in the inelastic domain, due to material elements that are unstable. In contrast, the modified GH model is expected to remain stable and encounter no convergence problems throughout the inelastic domain. In the second, the geometry and loading are identical to the first, but the laminate is quasi-isotropic. The $45^{\circ}$ stress-strain curve is modified to have a tangent modulus of $50 \mathrm{GPa}$ in the inelastic domain, so that $f_{45}=f_{0}$. As noted above, the modified and original models are expected to yield identical results for this material and, for both models, all material elements should be stable.

The finite element simulation was conducted in ABAQUS Standard (Version 6-9.2, Dassault Systèmes). The specimen geometry is depicted in Fig. 3. The plate width is five times the hole diameter. A quarter-symmetry finite element model is employed, with fournoded, quadrilateral, plane-stress elements. Load is applied in the $y$-direction (i.e. at $90^{\circ}$ ). A study was performed to ensure that the stresses and strains converged with respect to mesh density. For the quasi-isotropic material, the scissoring parameters $D_{0}$ and $D_{45}$ both equal 0 , by definition. For the inelastically anisotropic

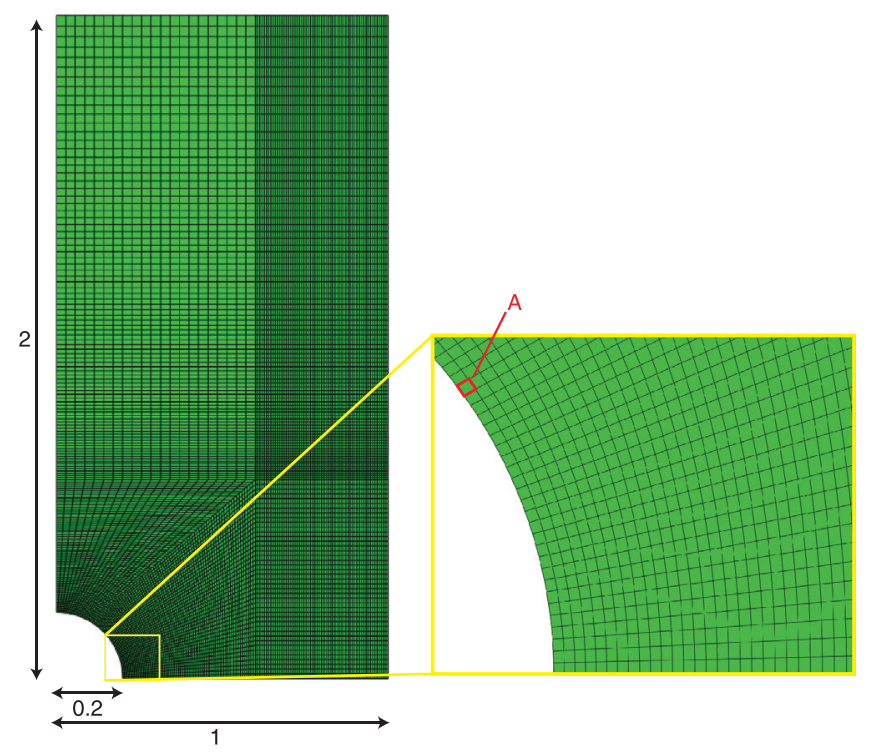

Fig. 3. Finite element mesh used to compute stress concentrations in open-hole tensile specimens. The element labeled $\mathrm{A}$ is the one interrogated to obtain the results in Fig. 6. (For interpretation of the references to color in this figure legend, the reader is referred to the web version of this article.) 
material, these parameters were set to 0 and 1 for the $0^{\circ}$ and $45^{\circ}$ directions, respectively. To explore the sensitivity of the results to $D_{45}$, a simulation was also run using the minimum value of this parameter that guaranteed stability $\left(D_{45}=0.76\right)$.

\subsection{Results}

For the quasi-isotropic laminate, stresses and strains in the direction of loading for the modified and original models are virtually identical to one another (within $0.05 \%$ ) in both the elastic and inelastic regimes, as expected. The computed stress concentration factor $k$ at the hole edge, normalized by that obtained in the elastic domain, $k_{e l}$, is plotted in Fig. $4 \mathrm{a}$ gainst the applied net-section stress, $\sigma_{\text {net }}$, normalized by the matrix cracking stress, $\sigma_{m c}$. The results show that stress redistribution due to inelasticity initially mitigates the stress concentration at the hole edge, thereby allowing the laminate to sustain larger loads before tensile fracture: a common feature in CMCs. More importantly, the stress concentration factors predicted by both models are identical. Furthermore, the tangential stiffness matrix for every element remains positive-definite throughout both simulations, and no convergence difficulties are encountered.

In contrast, for the anisotropic laminate, stresses and strains for the two models differ slightly, since the scissoring adjustment is present in the modified model but absent in the original model. Therefore, the stress concentration factors, shown in Fig. $4 \mathrm{~b}$, are also slightly different (by approximately $0.3-0.6 \%$ after net-section matrix cracking). The original model yields unstable behavior after matrix cracking. As a consequence, the solver eventually fails to

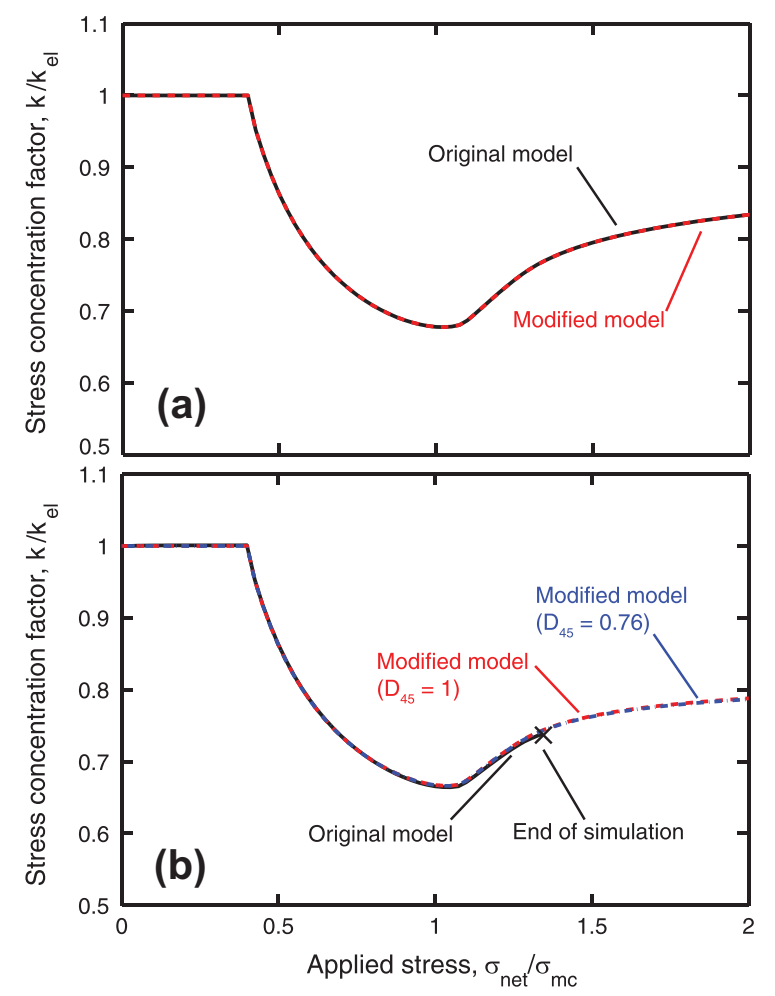

Fig. 4. Stress concentration factor at hole edge vs. applied stress for (a) the quasiisotropic material and (b) the inelastically-anisotropic material. Results for the original and modified GH models are shown. Two values of $D_{45}(0.76$ and 1$)$ are considered for the anisotropic material. The stress concentration factor is normalized by that in the elastic domain. The applied stress is computed on a net-section basis and is normalized by the matrix cracking stress. (For interpretation of the references to color in this figure legend, the reader is referred to the web version of this article.)

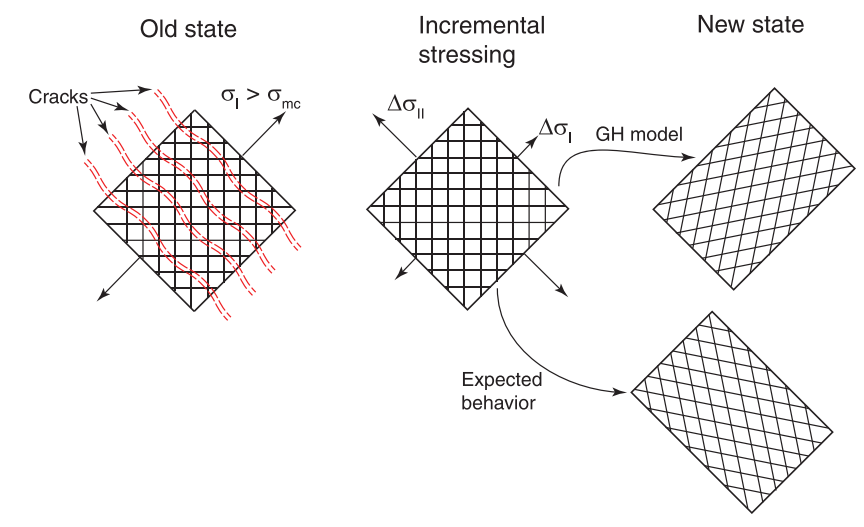

Fig. 5. Deformation of a cracked crossply laminate undergoing fiber scissoring. The behavior predicted by the GH model is shown at top right; that expected from consideration of fiber scissoring is shown at bottom right. (For interpretation of the references to color in this figure legend, the reader is referred to the web version of this article.)

obtain a solution; in the present case, this occurs at an applied stress of $\sigma_{\text {net }} / \sigma_{m c}=1.34$. The stress history for one unstable element (see Fig. 3) is plotted in Fig. 6. The stresses ( $\sigma_{y y}$, in the direction of loading) initially increase monotonically and smoothly; however, at $\sigma_{\text {net }} / \sigma_{m c}=1.15$, matrix cracking commences within the element. The tangential stiffness matrix loses positivedefiniteness shortly thereafter, causing the stresses to diverge and the stress history to lose monotonicity. The stress history for the same element in the modified model is also plotted in Fig. 6; the element is seen to exhibit stable behavior.

The simulation using the value $D_{45}=0.76$ yields results almost identical to those for $D_{45}=1$. The discrepancy between the axial stresses along the net-section symmetry plane (i.e. the incipient fracture plane) is very small: less than $0.2 \%$ for all values of applied stress. Results for the stress concentration factor for $D_{45}=0.76$ are shown in Fig. 4b. They indicate that the stress concentration factor is essentially independent of $D_{45}$. Physically, these results arise because, in notched tension simulations, the $0^{\circ}$ properties dominate the behavior, whereas the shear properties have a decidedly secondary effect. This fact can be verified by comparing the stress concentration factor for the quasi-isotropic material (in which the laminate is stiff in shear) with that for the anisotropic material (in which the laminate is compliant in shear after matrix cracking). Recall that the only effect of $D_{45}$ is to marginally alter the shear

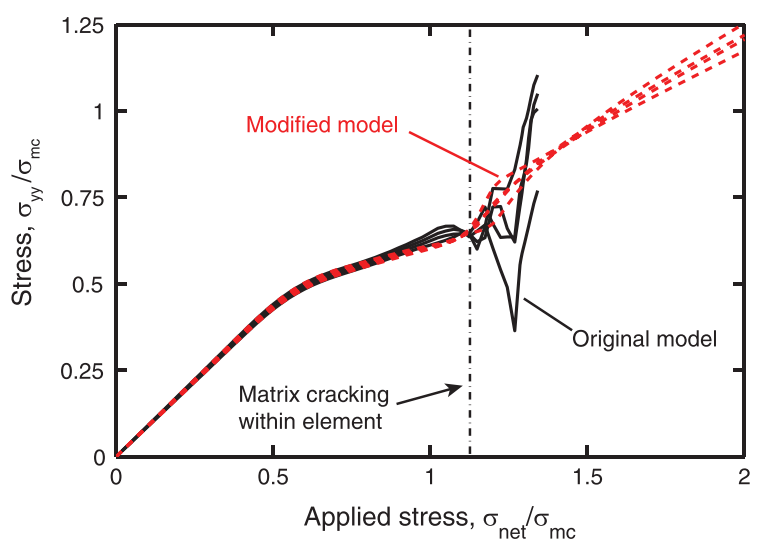

Fig. 6. Evolution of normal stress in direction of loading $\left(\sigma_{y y}\right)$ for one specific unstable element (indicated in Fig. 3 ) in the notched tension simulation. Stresses are plotted at all four integration points within the element. (For interpretation of the references to color in this figure legend, the reader is referred to the web version of this article.) 
compliance in the post-matrix cracking regime; $D_{45}$ leaves the $0^{\circ}$ response unchanged. The implication is that, for open-hole tension tests, $D_{45}$ can be set to unity without greatly affecting the accuracy of the results. However, for tests in which the shear properties predominate, the scissoring parameter must be selected more carefully.

The assumption of nearly proportional straining, used to derive the incremental model, was checked numerically for both the quasi-isotropic and anisotropic laminates. The orientation of the axes of principal strain $\left(\theta_{\epsilon}\right)$ was found to rotate by less than $0.1 \mathrm{rad}$ $\left(5.7^{\circ}\right)$ for almost all elements. In a few elements along the hole boundary, which experienced larger shear strains, $\theta_{\epsilon}$ rotated by a larger angle, with the maximum rotation angle being $0.35 \mathrm{rad}$ $\left(20^{\circ}\right)$. Therefore, the strains are nearly proportional for the vast majority of the laminate; the presence of mildly non-proportional straining in a small number of elements is surmised to negligibly affect the accuracy of the finite element analysis.

\section{Summary and conclusions}

We have demonstrated both analytically and by finite element analyses that the original formulation of the GH model is unstable for certain anisotropic laminates. Two distinct sources of instability have been identified: one associated with incremental shearing of elastically-anisotropic laminates and the other with incremental transverse straining of inelastically-anisotropic laminates. Both instabilities are manifested only in the post-matrix cracking regime. The sources of instability are more apparent in the incremental formulation of the model because the tangential stiffness matrix is explicitly present.

The instabilities are remedied by two proposed modifications. In the first, shear-extension coupling after matrix cracking is assumed to be negligible (even if it exists in the elastic domain). Although this assumption seems physically plausible, it remains to be validated experimentally. In the second, a modified tangential compliance matrix is developed in order to account for fiber scissoring, assuming simultaneous operation of two modes of deformation: axial fiber stretching and fragmentation (which was described in the original GH model) and fiber rotation (which was not). For this purpose, a scissoring parameter, $D_{\theta}$, is introduced to characterize the relative contributions of the two modes to the overall compliance. This parameter must equal 0 in the $0^{\circ}$ direction; in the $45^{\circ}$ direction, the value is 0 for quasi-isotropic laminates and close to 1 for $\left[0^{\circ} / 90^{\circ}\right]$ laminates. Although the precise value of $D_{45}$ for the latter laminates cannot be rationally selected, it appears to exert only a weak influence on the stress distribution in open-hole tension simulations. Most importantly, it yields stable behavior throughout. Thus, finite element analyses employing the modified model run stably whereas those with the original model encounter convergence problems. Future work will include an experimental assessment of the model as well as parametric studies to probe the effects of anisotropy on stress distributions in CMC laminates.

\section{Acknowledgements}

This work was supported by the Pratt \& Whitney Center of Excellence at the University of California, Santa Barbara (monitored by Douglas Berczik), and the US AFOSR (Ali Sayir) and NASA (Anthony Calomino) under the National Hypersonics Science Center for Materials and Structures (AFOSR Prime Contract No. FA9550-09-1-0477 to Teledyne Scientific and Sub-contract No. B9U538772 to UCSB). VPR was supported in part by a National Defense Science and Engineering Graduate Fellowship. The authors greatly acknowledge fruitful discussions with Guy Genin and John Hutchinson regarding this work.

\section{References}

[1] Genin GM, Hutchinson JW. Composite laminates in plane stress: constitutive modeling and stress redistribution due to matrix cracking. J Am Ceram Soc 1997;80(5):1245-55.

[2] McNulty JC, Zok FW, Genin GM, Evans AG. Notch-sensitivity of fiber-reinforced ceramic-matrix composites: effects of inelastic straining and volumedependent strength. J Am Ceram Soc 1999;82(5):1217-28.

[3] Hill R. A general theory of uniqueness and stability in elastic-plastic solids. J Mech Phys Solids 1958;6:239-49.

[4] Bažant ZP, Cedolin L. Stability of structures: elastic, inelastic, fracture and damage theories. Singapore: World Scientific Publishing Co. Pte. Ltd.; 2010.

[5] Maier G, Hueckel T. Nonassociated and coupled flow rules of elastoplasticity for rock-like materials. Int J Rock Mech Min Sci 1979;16(2):77-92.

[6] Bigoni D, Hueckel T. Uniqueness and localization - I. Associative and nonassociative elastoplasticity. Int J Solids Struct 1991;28(2):197-213.

[7] Bažant ZP. Comment on orthotropic models for concrete and geomaterials. J Eng Mech 1983;109(3):849-65.

[8] Curtin WA. Theory of mechanical properties of ceramic-matrix composites. J Am Ceram Soc 1991;74(11):2837-45.

[9] Hui CY, Phoenix SL, Ibnabdeljalil M, Smith RL. An exact closed form solution for fragmentation of Weibull fibers in a single filament composite with applications to fiber-reinforced ceramics. J Mech Phys Solids 1995;43(10):1551-85.

[10] Cady C, Heredia FE, Evans AG. In-plane mechanical properties of several ceramic-matrix composites. J Am Ceram Soc 1995;78(8):2065-78.

[11] Sloan SW, Abbo AJ, Sheng D. Refined explicit integration of elastoplastic models with automatic error control. Eng Computat 2001;18(1/2):121-54. 